\title{
6 A Surprisingly Puzzling Reality
}

Seldom do we pause to wonder how and why things work the way they do - they just are that way. However, scratch the thin surface of reality and it quickly becomes odd.

Here is a case in point.

Why does a football - an object that has exactly zero memory or consciousness of how it was kicked in the recent past, 'know' in which direction or with which force to go following that kick?

How does it independently 'know' the direction it should go and with which speed? Does it somehow possess a memory capability that it can tap and then use and act upon? (The answer to the latter question, as is expected, is a resounding no; the football has no clue whatsoever of how or if or when or with which energy or in which direction it was kicked, and has no capability to store any such memory in a useable or actionable way (29). How does it then obediently proceed in the direction it was kicked, after a direction-imparting event (the kick) which it cannot and does not remember?

To try and answer, let's imagine a thought experiment: some kind of futuristic operation has left you with vastly enhanced eyesight and extraordinary signalprocessing abilities within your brain to match. You are now able to see a snapshot of anything before you by batting your eyelids from a shut-eyes position back to a shuteyes position in less than a billionth of a second.

Someone fires a perfectly spherical bullet across your face when your eyes are shut, and you bat your eyelids.

What you catch a glimpse of is a perfect metal sphere hanging motionless before your eyes.

You have no means whatsoever of knowing where that bullet is going. You have absolutely no clue where the bullet is headed. Yet, you have infinitely more memory and more intelligence than the bullet itself.

How does the bullet know where to go?

The same of course applies to any projectile - to anything that was given an impulse at some time in the past. How does an inanimate object 'know' or 'remember' the direction it was fired, or for that matter the speed it is supposed to assume (e.g., imparted by the quantity of explosive in the cartridge)? How is any trajectory ever kept to?

The answer is astonishing. Indeed, the bullet or football or arrow does not know at all where it was fired or kicked to. It is simply not equipped to have the faintest clue that it was acted upon in any way.

The inescapable - and in fact correct - conclusion is that the object "recalculates" (for lack of a better word) where it should go (i.e., its trajectory) at every instant in time. How it quite does that, and exactly what 'every instant in time' may mean (what it is that triggers the instant of the recalculation) is not fully understood. The mathematical equation which describes the 'recalculation' that the object undergoes is, however, well known. 
This result has been proven both theoretically and experimentally. We will look at it more in depth in a minute.

But first, before we delve further into material reality and what weaves it into being or at the very least makes it tick, we must look again at ..... mathematics. 